\title{
Benefits of ambulatory axillary intra-aortic balloon pump for circulatory support as bridge to heart transplant
}

\author{
Ramanan Umakanthan, MD, Steven J. Hoff, MD, Natalia Solenkova, MD, Mark A. Wigger, MD, \\ Mary E. Keebler, MD, Andrew Lenneman, MD, Marzia Leacche, MD, Thomas G. DiSalvo, MD, \\ Henry Ooi, MD, Allen J. Naftilan, MD, John G. Byrne, MD, and Rashid M. Ahmad, MD
}

\begin{abstract}
Objective: Axillary intra-aortic balloon pump therapy has been described as a bridge to transplant. Advantages over femoral intra-aortic balloon pump therapy include reduced incidence of infection and enhanced patient mobility. We identified the patients who would benefit most from this therapy while awaiting heart transplantation.

Methods: We conducted a single-center, retrospective observational study to evaluate outcomes from axillary intra-aortic balloon pump therapy. These included hemodynamic parameters, duration of support, and success in bridging to transplant. We selected patients on the basis of history of sternotomy, elevated panel-reactive antibody, and small body habitus. Patients were made to ambulate aggressively beginning on postoperative day 1 .
\end{abstract}

Results: Between September 2007 and September 2010, 18 patients underwent axillary intra-aortic balloon pump therapy. All patients had the devices placed through the left axillary artery with a Hemashield side graft (Boston Scientific, Natick, Mass). Before axillary placement, patients underwent femoral placement to demonstrate hemodynamic benefit. Duration of support ranged from 5 to 63 days (median $=19$ days). There was marked improvement in ambulatory potential and hemodynamic parameters, with minimal blood transfusion requirements. There were no device-related infections. Some $72 \%$ of the patients $(13 / 18)$ were successfully bridged to transplantation.

Conclusions: Axillary intra-aortic balloon pump therapy provides excellent support for selected patients as a bridge to transplant. The majority of the patients were successfully bridged to transplant and discharged. Although this therapy has been described in previous studies, this is the largest series to incorporate a regimen of aggressive ambulation with daily measurements of distances walked. (J Thorac Cardiovasc Surg 2012;143:1193-7)

Cardiac transplantation is currently the definitive therapy for patients with end-stage heart failure. However, the availability of donor hearts to perform transplantation remains a major limiting factor. The United Network for Organ Sharing Registry data show the number of available hearts to range from 3000 to 3500 over the last several years. ${ }^{1}$ With an increasing number of patients developing endstage heart failure each year, the number awaiting cardiac transplantation far exceeds the number of available donor hearts.

As a result, mechanical support devices have been used increasingly as a bridge-to-transplant strategy to sustain patients' hemodynamics and organ function while they are on the heart transplant waiting list. Specific hemodynamic criteria are applied when the use of mechanical support is

From the Vanderbilt Heart and Vascular Institute, Nashville, Tenn.

Disclosures: Authors have nothing to disclose with regard to commercial support.

Received for publication Oct 30, 2011; revisions received Jan 13, 2012; accepted for publication Feb 3, 2012; available ahead of print Feb 27, 2012.

Address for reprints: Rashid M. Ahmad, MD, Department of Cardiac Surgery, Vanderbilt Heart and Vascular Institute, Nashville, TN 37232-8802 (E-mail:

rashid.ahmad@vanderbilt.edu).

$0022-5223 / \$ 36.00$

Copyright (C) 2012 Published by Elsevier Inc. on behalf of The American Association for Thoracic Surgery

doi:10.1016/j.jtcvs.2012.02.009 considered. These criteria include a cardiac index of less than $2.2 \mathrm{~L} / \mathrm{min} / \mathrm{m}^{2}$, systolic blood pressure of less than 90 $\mathrm{mm} \mathrm{Hg}$, mean pulmonary capillary wedge pressure or central venous pressure of greater than $20 \mathrm{~mm} \mathrm{Hg}$, and concomitant use of high doses of at least 2 inotropic agents. ${ }^{1} \mathrm{~A}$ patient's initial decline in hemodynamic status may first be managed pharmacologically with inotropes and an intra-aortic balloon pump (IABP), which is usually percutaneously inserted via the femoral artery. Although a femoral IABP can be inserted with relatively low risk and ease, major disadvantages include risk of infection, limited duration of support (3-7 days), and severe restriction of a patient's mobility. This constraint further deconditions a patient's musculoskeletal system. Insertion of an IABP via the axillary artery overcomes these disadvantages, and patients are able to ambulate.

Although there are a multitude of intermediate and longterm circulatory support devices available, the implantation of these devices can be costly financially and physiologically. In certain patients, the surgical risk may be high because of multiple previous cardiac surgeries. Some patients may also present with issues that render the use of these devices difficult, including the inability to tolerate higher levels of anticoagulation, history of sternotomies, elevated panel-reactive antibody (PRA) levels, or small 


\section{Abbreviations and Acronyms \\ BTT = bridge to transplant \\ IABP $=$ intra-aortic balloon pump \\ LVAD $=$ left ventricular assist device \\ PRA = panel-reactive antibody}

body habitus, which could make implantation difficult. The patient may have psychosocial issues that preclude the use of a left ventricular assist device (LVAD). Axillary IABP counterpulsation therapy is an alternate strategy in these scenarios, providing a feasible bridge-to-transplant option while maintaining patient mobility. In addition to containing cost, axillary IABP therapy also provides several other advantages compared with standard LVAD therapy. It is a less complicated surgical procedure with shorter extubation times and reduced blood transfusion requirements, and does not require specialized infrastructure and personnel for care as LVAD therapy does. Other distinct advantages with axillary IABP therapy include a low incidence of device-related infections, postoperative bleeding, stroke, and thrombosis.

We report our experience over a 2-year period in which axillary IABP support was used in 18 patients as a bridge to transplant (BTT). The patients underwent axillary IABP placement in our hybrid operating room, which combines the facilities of a cardiac surgery operating room with a cardiac catheterization laboratory.

\section{MATERIALS AND METHODS}

We conducted a retrospective review of the first 18 patients in whom left axillary IABPs were placed between September 2007 and 2010 at the Vanderbilt Heart Institute after obtaining institutional review board approval. All patients undergoing axillary IABP insertion had end-stage heart failure and failed ongoing inotropic support or could not tolerate inotropic therapy because of arrhythmias from the inotropes. We specifically identified patients with a history of sternotomy, elevated PRA $(>20 \%)$, or small body habitus (body mass index $<25$ ). Before selection for axillary IABP placement, patients underwent femoral balloon placement to establish hemodynamic benefit from counterpulsation therapy ranging from improved cardiac output and reduced pulmonary artery pressures.

Our management plan was to wean the femoral IABP, and if this was tolerated, the femoral IABP was taken out the same day before axillary IABP insertion. If the wean was not tolerated, then the wire was already in place for the axillary IABP before taking out the femoral IABP in the operating room. We were cautious in ascertaining that the hemodynamic benefit from the axillary IABP was similar to the femoral test insertion.

All patients were brought to the operating room, and monitoring lines were placed. Preoperative intravenous prophylactic antibiotics were given. After induction, the patient was prepped and draped in the standard fashion. An incision was made below the left clavicle. The fibers of pectoralis major and minor were split. The fascia was then incised with Metz scissors. Vessel loops were placed around the axillary vein and artery. Heparin was given, and the axillary artery was clamped proximally and distally. An appropriate arteriotomy was made, and a 6-mm Hemashield graft (Boston Scientific, Natick, Mass) was sewn with 5-0 running Prolene. The graft was allowed to back-bleed, and good hemostasis was achieved.
TABLE 1. Patient characteristics

\begin{tabular}{lc}
\hline \multicolumn{1}{c}{ Variables } & Patients (n=18) \\
\hline Median age (y) & $56(20-64)$ \\
Gender & \\
Male & $15 / 18(93 \%)$ \\
Female & $3 / 18(17 \%)$ \\
BMI $\left(\mathrm{m}^{2} / \mathrm{kg}\right)$ & $25.55(19.66-33.22)$ \\
Diabetes & $7 / 18(39 \%)$ \\
Hypertension & $7 / 18(39 \%)$ \\
COPD (moderate to severe) & $3 / 18(16.7 \%)$ \\
Creatinine $\geq 1.5$ mg/dL at the time of & $7 / 18(39 \%)$ \\
$\quad$ IABP insertion & \\
Chronic renal failure on hemodialysis & $1 / 18(5.6 \%)$ \\
Inotropic support & $18 / 18(100 \%)$ \\
Median ejection fraction $(\%)$ & $15(5-25)$ \\
Congestive heart failure & $18 / 18(100 \%)$ \\
Mean NYHA class & Class IV \\
Carotid artery disease with $>75 \%$ stenosis & $3 / 18(16.7 \%)$ \\
Prior myocardial infarction & $3 / 18(16.7 \%)$ \\
Atrial fibrillation & $7 / 18(39 \%)$ \\
Previous sternotomy or elevated PRA $(>20 \%)$ & $10 / 18(56 \%)$ \\
$\quad$ or small body habitus (BMI $<25)$ & \\
Ischemic cardiomyopathy & $9 / 18(50 \%)$ \\
Dilated cardiomyopathy & $9 / 18(50 \%)$ \\
Mitral insufficiency (moderate to severe) & $7 / 18(39 \%)$ \\
Tricuspid insufficiency (moderate to severe) & $5 / 18(27.8 \%)$ \\
\hline Vas prested a mo & \\
\hline
\end{tabular}

Values presented as mean, median (range), or number (percent). BMI, Body mass index; $C O P D$, chronic obstructive pulmonary disease; IABP, intra-aortic balloon pump; NYHA, New York Heart Association; PRA, panel-reactive antibody.

The free end of the graft was shortened and oversewn with running 5-0 Prolene suture.

A pledgeted purse-string with 5-0 Prolene was placed on the anterior surface of the graft. A needle was placed in the center of the pursestring, and the balloon wire was advanced. This was done using fluoroscopy.

The entry site was successively dilated. Once the wire was confirmed to be in the descending aorta, the IABP was advanced over the wire into the descending aorta. Care was taken to flush and de-air the lines. The IABP was initiated, and positioning was reconfirmed by transesophageal echocardiography.

After confirmation of hemostasis, the axillary incision was closed in layers. The stump of the graft was placed superficial to the pectoralis major fascia. The skin was approximated with running Monocryl suture. The IABP was secured to the skin.

Patients were returned to the cardiovascular intensive care unit in stable condition. Aggressive ambulatory therapy was initiated from the first postoperative day. Parameters such as cardiac index, mean and systolic pulmonary artery pressures, central venous pressure, and maximum distance ambulated daily were recorded and compared with their respective values pre-IABP placement. Time to extubation, blood transfusion requirements, and changes in creatinine were also recorded. The duration of support on the IABP and time to cardiac transplant (as appropriate) were recorded. A 2-tailed paired $t$ test was used for statistical comparison of pre- and post-IABP parameters.

\section{RESULTS}

Between September 2007 and 2010, 18 patients were managed with axillary IABP therapy at the Vanderbilt Heart 
TABLE 2. Perioperative data

\begin{tabular}{lccrr}
\hline & $\begin{array}{c}\text { Pre-IABP } \\
\mathbf{n}=\mathbf{1 8}\end{array}$ & $\begin{array}{c}\text { Post-IABP } \\
\mathbf{n}=\mathbf{1 8}\end{array}$ & $\boldsymbol{P}$ value & Percent improvement \\
\hline CI & $1.91 \pm 0.48$ & $2.84 \pm 0.70$ & $<.001$ & .025 \\
PA systolic & $54.65 \pm 17.77$ & $45.60 \pm 11.89$ & .009 & 49 \\
PA mean & $38.82 \pm 12.40$ & $31.07 \pm 9.35$ & $<.001$ & .008 \\
CVP & $24.12 \pm 8.44$ & $12.65 \pm 5.93$ & \\
Longest distance walked/day (ft) & $411.4 \pm 247.7$ & $2654.58 \pm 2425.52$ & \\
PRBC 24 h postoperatively & & $0.11 \pm 0.32$ & \\
Time to extubation in hours & & $0.64 \pm 1.65$ & \\
Survival & & & \\
$\quad 1$ mo & & $16 / 18(89 \%)$ & \\
$\quad 6$ mo & & $13 / 18(72 \%)$ & \\
No. transplanted & & $13 / 18(72 \%)$ & \\
Days to transplant & & $26.7 \pm 17.7$ & \\
& & Median: 19 & \\
& & Range: $5-63$ & \\
\hline
\end{tabular}

Values presented as mean (standard deviation), median (range), or number (percent). IABP, intra-aortic balloon pump; $C I$, cardiac index; $P A$, pulmonary artery; $C V P$, central venous pressure; $P R B C$, packed red blood cells.

Institute as BTT. Patient characteristics are shown in Table 1. Fifty percent of the patients presented with ischemic cardiomyopathy, and $50 \%$ of the patients presented with dilated cardiomyopathy. Fifty-six percent of the patients $(10 / 18)$ had a history of sternotomy or elevated PRA levels $(>20 \%)$ or small body habitus (body mass index $<25$ ).

All patients had ambulatory IABPs successfully placed through the left axillary route. The median time to extubation was $0.64 \pm 1.65$ hours. Sixty-seven percent (12/18) of the patients were extubated in the operating room.

After axillary IABP placement, there was significant hemodynamic response (Table 2). Specifically, we observed improvements in cardiac index $(2.84 \pm 0.70$ post-IABP vs $1.91 \pm 0.48$ pre-IABP, $P=.001$ ), decrease in mean pulmonary arterial pressure $(31.1 \pm 9.4 \mathrm{~mm} \mathrm{Hg}$ post-IABP vs 38.8 $\pm 12.4 \mathrm{~mm} \mathrm{Hg}$ pre-IABP, $P=.009$ ), and decrease in central venous pressure $(12.7 \pm 5.9 \mathrm{~mm} \mathrm{Hg}$ post-IABP vs $24.1 \pm$ $8.4 \mathrm{~mm} \mathrm{Hg}$ pre-IABP, $P=.001)$. The duration of

TABLE 3. Adverse events

\begin{tabular}{ll}
\hline \multicolumn{1}{c}{ Complication } & Patients $(\mathbf{n}=\mathbf{1 8})$ \\
\hline IABP-related infection & $0 / 18(0 \%)$ \\
IABP failure & $3 / 18(17 \%)$ \\
$\quad$ Migration & $1 / 18(5.6 \%)$ \\
Kinking & $1 / 18(5.6 \%)$ \\
$\quad$ Rupture & $1 / 18(5.6 \%)$ \\
Mortality before transplantation & $5 / 18(27.8 \%)$ \\
$\quad$ Multisystem organ failure from pulmonary & $2 / 18(11.2 \%)$ \\
$\quad$ infection & \\
Myocardial infarction & $1 / 18(5.6 \%)$ \\
Progressive heart failure & $1 / 18(5.6 \%)$ \\
Cardiac arrest & $1 / 18(5.6 \%)$ \\
IABP-related vascular complication & $0 / 18(0 \%)$ \\
Stroke & $0 / 18(0 \%)$ \\
Other miscellaneous complications & $0 / 18(0 \%)$ \\
\hline Val
\end{tabular}

Values presented as number (percent). IABP, intra-aortic balloon pump. ambulatory IABP support ranged from 5 to 63 days (26.7 \pm 17.7 days), and the duration of time to transplant was $26.7 \pm 17.7$ days. The longest distance walked in a day was $2654.58 \pm 2425.52$ feet post-IABP vs $411.4 \pm 247.7$ feet pre-IABP $(P=.008)$. The majority of patients $(72 \%$, 13/18) underwent successful cardiac transplantation and were discharged from the hospital.

Major complications of axillary IABP support in this series are shown in Table 3. Three patients required IABP replacement because of device migration, kinking, and rupture. There were no deaths in this group. Mean blood transfusion requirement 24 hours after IABP placement was $0.11 \pm 0.32$ units. Only 2 patients in the series required blood transfusions. Five patients died on axillary IABP support while waiting for cardiac transplant. Two patients died of multisystem organ failure secondary to pulmonary infection, 1 patient died of a massive myocardial infarction, 1 patient died of progressive heart failure, and 1 patient died of cardiac arrest. Figures 1 and 2 show bar graph comparisons of pre-IABP and post-IABP cardiac index and comparisons of longest distance walked in 1 day.

\section{DISCUSSION}

In our experience, we found that axillary IABP therapy in selected patients provides hemodynamic support, maintains excellent mobility, and has a BTT rate of $72 \%$. It is a less complicated surgical procedure with a shorter period of ventilator support and reduced blood transfusion requirements. It is specifically useful in patients with a history of sternotomy, elevated PRA, and small body habitus. In addition, there is a low incidence of device-related infections, postoperative bleeding, stroke, and thrombosis. Axillary IABP therapy should be considered in the armamentarium for strategic mechanical circulatory support in appropriately selected patients. 


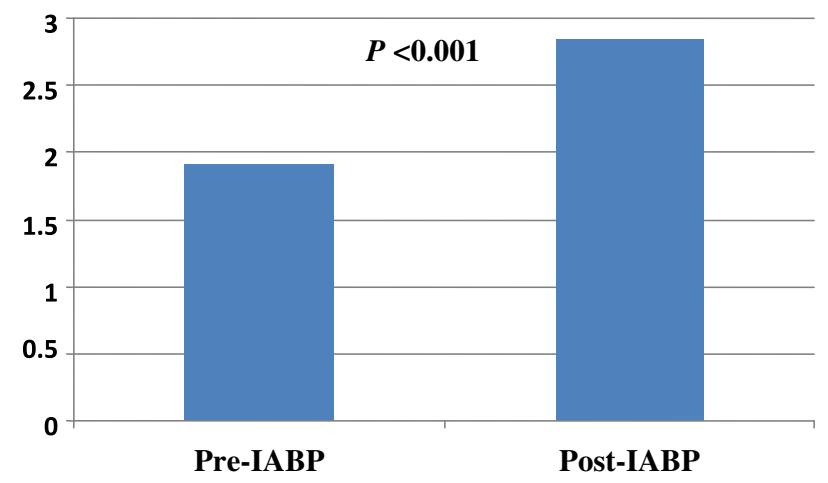

FIGURE 1. Bar graph comparison of pre-IABP and post-IABP cardiac index. $I A B P$, Intra-aortic balloon pump.

The incidence of end-stage heart failure continues to increase, whereas the availability of donor hearts remains a fixed resource. Because of the shortage of donor hearts, alternate solutions have been pursued for hemodynamic stabilization and optimization. The literature shows implantation of a ventricular assist device as BTT has significant risks, such as postoperative bleeding, stroke, blood transfusions, and drive-line infections leading to sepsis. In addition, the financial costs of ventricular assist device therapy should be considered, especially in the current era of cost-consciousness. Therefore, cost containment, efficacy, and postoperative complications of mechanical support must be considered in BTT. Future studies will be necessary to assess axillary IABP strategy in the context of ventricular assist device therapy.

The first insertion of the IABP via the axillary approach was described by McBride and colleagues ${ }^{2}$ in 1989. Thereafter, H'Doubler and colleagues ${ }^{3}$ published the largest series in 2000 consisting of 13 patients who underwent axillary IABP therapy over the course of a 3-year period. Of these, 10 patients were successfully bridged to transplant with duration of IABP support ranging from 10 to 86 days. Subsequently in 2002, Cochran and colleagues ${ }^{4}$ published the results of their series on 4 patients who underwent axillary IABP therapy over the course of a 1.5 -year period. All patients were successfully bridged to transplant, and the duration of IABP support ranged from 12 to 70 days.

Our series, the largest so far, indicates that ambulatory IABPs placed via the axillary route in selected patients provide excellent support as BTT without hindering mobility in selected patients. It also demonstrates that aggressive ambulatory therapy initiated promptly after axillary IABP insertion can prove to be of tremendous benefit. There were no device-related infections or bleeding or embolic complications. Patients were extubated in the operating room or on arrival to the cardiovascular intensive care unit without any complications. There were 5 mortalities, and these were not device related. There were no complications involving the axillary artery or the left upper extremity.

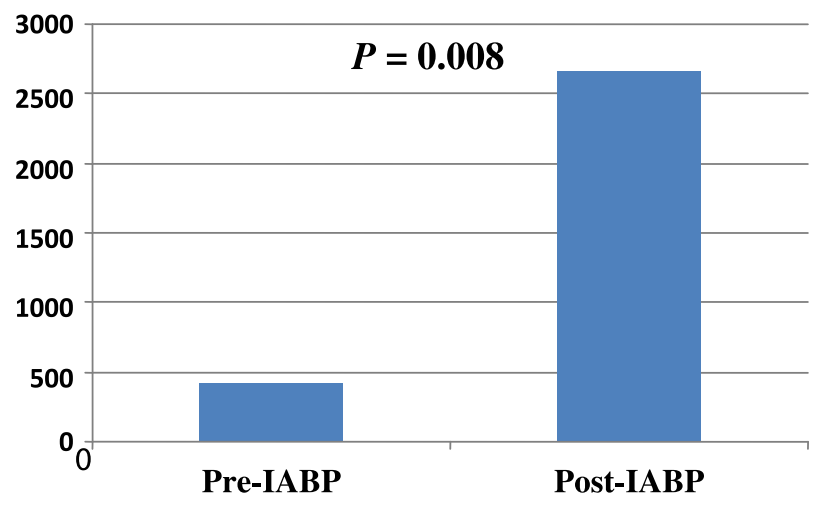

FIGURE 2. Bar graph comparison of longest distance walked in 1 day (in feet) pre-IABP and post-IABP. IABP, Intra-aortic balloon pump.

Of these mortalities, the 1 patient who subsequently received biventricular support was a complex case. In addition to heart failure, this patient had a severe lowerextremity infection, and we were hoping to control this infection and avoid implantation of excess hardware. As the patient's condition worsened from a circulatory standpoint, we had to increase the level of support. If this patient did not have lower-extremity cellulites, he would have gone straight to long-term mechanical support. This patient was transitioned to a biventricular assist device but ultimately died as the result of worsening of infection and subsequent pulmonary infection-related multisystem organ failure. A second patient requested removal of circulatory support and subsequently died of heart failure. A third patient died with subsequent pulmonary infection and multisystem organ failure after the family decided to withdraw care by the family. A fourth patient died after nonischemic cardiomyopathy resulting from a massive myocardial infarction. The fifth patient died of sudden arrhythmia and cardiac arrest. All mortalities also occurred in cases of nonischemic cardiomyopathy. Thus, the benefit of this technique in nonischemic patients may be less than in ischemic patients. In general, our criteria were to use this less-invasive technique of axillary IABP placement in patients who have demonstrated benefit from femoral IABP placement. If we observed that the axillary IABP did not improve the indices of perfusion and hemodynamic parameters, the plan was to transition to ventricular assist device support. As mentioned, only 1 patient required transition to ventricular assist device support.

All the mortalities were in nonischemic patients. Thus, our study seems to also indicate that this technique is more beneficial in ischemic patients. This may be a reflection of nonischemic patients receiving intervention at a physiologically worse point on their heart failure curve. This warrants further investigation.

There are many advantages to an ambulatory axillary IABP therapy over LVAD placement. In addition to reduced 
device-related infections, stroke, thrombosis, postoperative bleeding, blood transfusion requirements, extubation times, and cost, the placement of an axillary IABP is a simpler procedure. Because the transfusion requirements are essentially nonexistent for IABP placement, the risk of increasing PRAs is virtually zero from the procedure. Moreover, Cochran and colleagues ${ }^{4}$ showed no evidence of elevated PRAs secondary to long-term IABP therapy.

Unlike LVAD insertion, placement of an axillary IABP does not require cardiopulmonary bypass or expose the patient to an additional sternotomy before heart transplantation. In addition, the removal of the axillary IABP is a relatively simple procedure at the time of cardiac transplantation. Furthermore, the axillary IABP also provides support while weaning from cardiopulmonary bypass. LVAD removal can be potentially difficult with bleeding complications. There were no thromboembolic events or any incidents of platelet dysfunction noted as a result of axillary IABP therapy in our series.

Every heart center has an ancillary infrastructure and personnel who are well versed in the management of an IABP. Excellent nursing experience with IABP management is well established in cardiac units. Therefore, expanding its use to the telemetry unit can be considered.

\section{Study Limitations}

Study limitations include that this was not a randomized prospective evaluation of the technology. In addition, the nutritional status of patients when they are bridged to transplant after axillary IABP placement was not evaluated.
Axillary IABP therapy has the limitation of requiring a prolonged intensive care unit stay. This constraint could be mitigated with appropriate training of the personnel and infrastructure in the step-down unit.

\section{CONCLUSIONS}

Our data show that axillary IABP therapy is an excellent means of conferring hemodynamic support in well-selected patients with end-stage heart failure with a successful BTT rate of $72 \%$. The axillary IABP enables patients' ambulation and optimization of rehabilitation potential. The axillary IABP improves hemodynamics and right ventricular performance, which may reduce the need for right ventricular assist device support during LVAD insertion if the patient does not receive a donor heart during the extended hospitalization. Clearly, future investigations will be necessary to determine optimal use of the axillary IABP in selecting patients for BTT versus the subset who do not receive transplants but become better candidates for LVAD therapy after optimization of right ventricular hemodynamics.

\section{References}

1. McGee EC Jr, McCarthy PM, Moazami N. Temporary mechanical circulatory support. In: Cohn LH, ed. Cardiac Surgery in the Adult. New York: McGraw-Hill; 2008. p. 507-34.

2. McBride LR, Miller LW, Naunheim KS, Pennington DG. Axillary artery insertion of an intra-aortic balloon pump. Ann Thorac Surg. 1989;48:874-5.

3. H'Doubler PB, H'Doubler WZ, Bien RC, Jansen DA. A novel technique for intraaortic balloon pump placement via the left axillary artery in patients awaiting cardiac transplantation. Cardiovasc Surg. 2000;8:463-5.

4. Cochran RP, Starkey TD, Panos AL, Kunzelman KS. Ambulatory intraaortic balloon pump use as bridge to heart transplant. Ann Thorac Surg. 2002;74:746-52. 